\title{
On Solvents of Matrix Polynomials
}

\author{
Malika Yaici and Kamel Hariche
}

\begin{abstract}
This paper is concerned with the construction of right and left solvents (also called block roots) of a matrix polynomial from latent roots and vectors. It addresses also the important case of the existence of a complete set of block roots. Solvents do not always exist, so conditions for the existence of such solvents are discussed. The inverse of a matrix polynomial is obtained as a particular case of the block partial fraction expansion of a related rational matrix. It involves the knowledge of a complete set of solvents and the computation of the inverse of a block Vandermonde matrix. Numerical examples are given to illustrate the two results.
\end{abstract}

Index Terms-Block partial fraction expansion, latent roots, latent vectors, matrix fraction description, matrix polynomials, solvents.

\section{INTRODUCTION}

Matrix polynomials play a central role in the mathematical description of the dynamics of multivariable systems. In this case MIMO systems are described by a matrix transfer function $G(s)$ expressed as a right (or left) matrix fraction description (RMFD or LMFD):

$$
\left\{\begin{array}{c}
G(s)=N_{r}(s) D_{r}^{-1}(s) \\
\text { or } \\
G(s)=D_{l}^{-1}(s) N_{\mathrm{l}}(s)
\end{array}\right.
$$

where $N_{r} D_{r} N_{1} D_{1}$ are matrix polynomials. This fact has led to an active research effort in matrix polynomials theory.

A theoretical introduction based on spectral approach of matrix polynomial theory is given in [1]; the same authors proposed an algorithm based on Jordan chains for the computation of a solvent of a matrix polynomial. Methods and algorithms for numerical solutions of spectral problems for one- and two-parameter polynomial and rational matrices are given in [2]. Some mathematical theory of $\lambda$ matrices, in particular solvents and interpolating $\lambda$-matrices are discussed in [3]. In this paper, the necessary and sufficient conditions for the existence of solvents and the corresponding generalized Lagrange interpolating $\lambda$ matrices are given.

Computing the inverse of a matrix polynomial is very important in all the fields dealing with matrix polynomials and has been treated by many researchers. Zhang [4] addressed the case of column reduced polynomial matrices to obtain an irreducible inverse. Schuster and Hippe [5] computed polynomial matrix inverses by interpolation, but the efficiency of the proposed algorithm is very dependent

Manuscript received January 10, 2014; revised May 15, 2014.

M. Yaici is with the Computer Department, University of Bejaia, Bejaia 06000, Algeria (e-mail: yaici_m@ hotmail.com).

$\mathrm{K}$. Hariche is with Electronics and Electronics Institute, University of Boumerdes, Boumerdes 35000, Algeria (e-mail: khariche@yahoo.com). on the interpolating points which are to be chosen.

An appropriate Sylvester resultant matrix has been used by Stefanidis et al., [6] to generate a real matrix to compute its inverse. In [7] an algorithm to obtain a minimal statespace representation is given then Leverrier's algorithm is used to determine the transfer function representation of the inverse. In [8] the case of the generalized inversion of a polynomial matrix is treated and an algorithm to compute the inverse is proposed.

Many authors have considered using matrix polynomial s and block roots for solving some linear algebra problems or control problems such as: compensator design using numerical optimization techniques [9], block partial fraction expansion of a matrix fraction description (MFD) with single and repeated poles [10], reduction of the order of single-input single-output and multi-input multi-output robust controllers [11], feedforward compensator in twodegree-of-freedom controllers [12] etc.

In this paper, we will consider r-degree, $m^{\text {th }}$ order monic matrix polynomials of the form

$$
D(\lambda)=I_{m} \lambda^{r}+D_{1} \lambda^{r-1}+\cdots+D_{r-1} \lambda+D_{r}
$$

where $\lambda$ is a complex number and $D_{i} \in \mathbb{R}^{m \times m}$.

A latent root $\lambda_{i}$ of $D(\lambda)$ is a complex number satisfying det $D\left(\lambda_{i}\right)=0$.

A right latent vector $X_{i} \in \mathbb{R}^{m}$ associated with $\lambda_{i}$ satisfies $D\left(\lambda_{i}\right) X_{i}=\theta$ while a left latent vector $Y_{i}$ is a row vector satisfying $Y_{i} D\left(\lambda_{i}\right)=\theta(\theta$ being used here to indicate the zero vector).

A right solvent of $D(\lambda)$ is an $\mathrm{m} \times \mathrm{m}$ matrix $R$ such that

$$
R^{r}+D_{1} R^{r-1}+\cdots+D_{r-1} R+D_{r}=0_{m}
$$

while a left solvent is an $\mathrm{m} \times \mathrm{m}$ matrix $L$ satisfying

$$
L^{r}+L^{r-1} D_{1}+\cdots+L D_{r-1}+D_{r}=0_{m}
$$

The purpose of this paper is to show that right and left solvents can be constructed from latent roots and corresponding right and left latent vectors. It follows that each of the block roots contains a part of the latent structure of the matrix polynomial.

A particular case of interest is the one where we can construct a set of block roots covering all the latent structure of $D(\lambda)$. This set is referred to as a complete set of block roots. The existence of right and left solvents is discussed in the paper as well as the existence of a complete set. Furthermore, the inverse $D^{-1}(\lambda)$ is given as a particular case of the block partial fraction expansion of a related rational matrix, this result being the main novelty of the paper.

The paper is organized as follows: after an introduction to the most important facts on matrix polynomials and lambdamatrices, a section is dedicated to the construction of 
right/left solvents from latent roots and latent vectors along with the condition of their existence. A section dealing with the block partial fraction expansion of matrix polynomials is given followed by the determination of $D^{-1}(\lambda)$. Finally a conclusion and some perspectives finish the paper.

\section{TheORETICAL PRELIMINARIES}

An $m^{\text {th }}$ order $r^{\text {th }}$ degree $\lambda$-matrix is given by:

$$
D(\lambda)=D_{0} \lambda^{r}+D_{1} \lambda^{r-1}+\cdots+D_{r-1} \lambda+D_{r}
$$

where $D_{i}(i=0 \ldots r)$ are $m \times m$ complex matrices and $\lambda$ a complex number.

For an $m \times m$ complex matrix $X$ we define the following: A right polynomial matrix is given by:

$$
D_{R}(X)=D_{0} X^{r}+D_{1} X^{r-1}+\cdots+D_{r-1} X+D_{r}
$$

A left polynomial matrix is given by:

$$
D_{L}(X)=X^{r} D_{0}+X^{r-1} D_{1}+\cdots+X D_{r-1}+D_{r}
$$

If $D_{0}$ is an identity matrix $I_{m}$ then the matrix polynomial is said to be monic.

If $D_{0}$ is singular and det $D(\lambda) \neq 0$ for any $\lambda$ then $D(\lambda)$ can be reversed to make $D_{0}$ non-singular.

\section{A. Latent Roots and Latent Vectors}

A latent root $\lambda_{\mathrm{i}}$ of $D(\lambda)$ is a complex number satisfying det $D\left(\lambda_{i}\right)=0$; A right latent vector $X_{i} \in \mathbb{R}^{m}$ associated with $\lambda_{\mathrm{i}}$ satisfies $D\left(\lambda_{\mathrm{i}}\right) X_{i}=\theta$; while a left latent vector $Y_{i}$ is a row vector satisfying $Y_{i} D\left(\lambda_{i}\right)=\theta$.

If $D(\lambda)$ has a singular leading coefficient $\left(D_{0}\right)$ then $D(\lambda)$ has latent roots at infinity.

\section{B. Solvents}

Right and left solvents are defined in equations 3 and 4. The following are important facts on Solvents [14]:

1) Solvents of a $\lambda$-matrix do not always exist.

2) If $R$ is a right solvent then there exists a $\lambda$-matrix $Q$ of degree $\mathrm{r}-1$ such that $D(\lambda)=Q(\lambda)(I \lambda-R)$.

3 ) If $\mathrm{L}$ is a left solvent then there exists a $\lambda$-matrix $S$ of degree $\mathrm{r}-1$ such that $D(\lambda)=(I \lambda-L) S(\lambda)$.

4) Generalized right (left) eigenvectors of a right (left) solvent are the generalized latent vectors of $D(\lambda)$.

$5) \mathrm{W}$ is a weak solvent if $D(W)$ is singular.

\section{Construction of Matrix Coefficients of a $\lambda$-Matrix}

A polynomial matrix can be determined (or its matrix coefficients) from a set of solvents under some conditions [9]

For each right solvent $R_{\mathrm{j}}(j=1 . . r)$ of $D(\lambda)$ we have: $R_{j}^{r}+D_{1} R_{j}^{r-1}+\cdots+D_{r-1} R_{j}+D_{r}=0_{m}$, then $D_{1} R_{j}^{r-1}+$ $\cdots+D_{r-1} R_{j}+D_{r}=-R_{j}^{r}$ or for $\mathrm{j}=1 \ldots r$.

$$
\left[\begin{array}{llll}
D_{r} & D_{r-1} & \cdots & D_{1}
\end{array}\right]\left[\begin{array}{c}
I_{m} \\
R_{j} \\
\vdots \\
R_{j}^{r-1}
\end{array}\right]=-R_{j}^{r}
$$

Then:

$$
\left[\begin{array}{llll}
D_{r} & D_{r-1} & \cdots & D_{1}
\end{array}\right]=-\left[\begin{array}{llll}
R_{1}^{r} & R_{2}^{r} & \cdots & R_{r}^{r}
\end{array}\right] V_{R}^{-1}
$$

where $V_{R}$ is a "right" block Vandermonde matrix:

$$
V_{R}=\left[\begin{array}{cccc}
I_{m} & I_{m} & \cdots & I_{m} \\
R_{1} & R_{2} & \cdots & R_{r} \\
\vdots & \vdots & \cdots & \vdots \\
R_{1}^{r-1} & R_{2}^{r-1} & \cdots & R_{r}^{r-1}
\end{array}\right]
$$

For each left solvent $L_{j}(j=1 . . r)$ of $D(\lambda)$ we have:

$$
L_{j}^{r}+L_{j}^{r-1} D_{1}+\cdots+L_{j} D_{r-1}+D_{r}=0_{m}
$$

Then following the same development:

$$
\left[\begin{array}{c}
D_{r} \\
D_{r-1} \\
\vdots \\
D_{1}
\end{array}\right]=-V_{L}^{-1}\left[\begin{array}{c}
L_{1}^{r} \\
L_{2}^{r} \\
\vdots \\
L_{r}^{r}
\end{array}\right]
$$

where $V_{L}$ is a block Vandermonde matrix:

$$
V_{L}=\left[\begin{array}{cccc}
I_{m} & L_{1} & \cdots & L_{1}^{r-1} \\
I_{m} & L_{2} & \cdots & L_{2}^{r-1} \\
\vdots & \vdots & \vdots & \vdots \\
I_{m} & L_{r} & \cdots & L_{r}^{r-1}
\end{array}\right]
$$

Theorem 1: A block Vandermonde matrix constructed from a complete set of solvents of a particular matrix polynomial is non singular

Proof 1: see [13].

\section{CONSTRuction OF SOlvents}

\section{A. Construction of Right Solvents}

Suppose that the set $\left\{\lambda_{1}, \lambda_{2} \ldots \lambda_{m}\right\}$ of $m$ latent roots of $\mathrm{D}(\lambda)$ has a linearly independent set of corresponding right latent vectors $\left\{X_{1}, X_{2}, \ldots X_{m}\right\}$. Let $M=\left(X_{1} X_{2} \ldots X_{m}\right)$ be the $m \times m$ matrix whose columns are the linearly independent right latent vectors and $M^{-1}=\left(\begin{array}{c}Y_{1} \\ Y_{2} \\ \vdots \\ Y_{m}\end{array}\right)$ be it inverse

It follows that $\sum_{i=1}^{m} X_{i} Y_{i}=I_{m}$ and $X_{i} Y_{j}=\delta_{i j}$ for $i, j=$ 1...m (from $\left.M M^{-1}=M^{-1} M=I_{m}\right)$.

Theorem 2: The $m \times m$ matrix $R=M \Lambda M^{-1}$, where $\Lambda=\operatorname{diag}$ $\left(\lambda_{1}, \lambda_{2}, \ldots \lambda_{\mathrm{m}}\right)$, is a right solvent of $D(\lambda)$.

Proof 2: Let $D_{R}(R)=R^{r}+D_{1} R^{r-1}+\cdots+D_{r-1} R+D_{r}$ Hence $D_{R}(R)$ can be rewritten as:

$$
\begin{gathered}
D(R)=M \Lambda^{r} M^{-1}+D_{1} M \Lambda^{r-1} M^{-1}+ \\
\ldots+D_{r-1} M \Lambda M^{-1}+D_{r}
\end{gathered}
$$

It can easily be verified that $M \Lambda^{l} M^{-1}=\sum_{i=1}^{m} \lambda^{l} X_{i} Y_{i} 1=$ $1 \ldots r$ which gives

$$
\begin{aligned}
D(R)= & \sum_{i=1}^{m} \lambda^{r} X_{i} Y_{i}+D_{1} \sum_{i=1}^{m} \lambda^{r-1} X_{i} Y_{i} \\
& +\cdots+D_{r-1} \sum_{i=1}^{m} \lambda X_{i} Y_{i}+D_{r}
\end{aligned}
$$

or

$$
\begin{gathered}
D(R)=\left(\lambda_{1}^{r} X_{1}+\lambda_{1}^{r-1} D_{1} X_{1}+\cdots+\lambda_{1} D_{r-1} X_{1}\right) Y_{1}+ \\
\left(\lambda_{2}^{r} X_{2}+\lambda_{2}^{r-1} D_{1} X_{2}+\cdots+\lambda_{2} D_{r-1} X_{2}\right) Y_{2}+\cdots+ \\
\left(\lambda_{m}^{r} X_{m}+\lambda_{m}^{r-1} D_{1} X_{m}+\cdots+\lambda_{m} D_{r-1} X_{m}\right) Y_{m}+D_{r}
\end{gathered}
$$

Since $\left\{\lambda_{i}, X_{i}\right\}$ is a latent pair it follows that: 


$$
\lambda_{i}^{r} X_{i}+\lambda_{i}^{r-1} D_{1} X_{i}+\cdots+\lambda_{i} D_{r-1} X_{i}+D_{r} X_{i}=\theta
$$

Or

$$
\lambda_{i}^{r} X_{i}+\lambda_{i}^{r-1} D_{1} X_{i}+\cdots+\lambda_{i} D_{r-1} X_{i}=-D_{r} X_{i}
$$

Leading to

$$
\begin{gathered}
D(R)=-D_{r} X_{1} Y_{1}-D_{r} X_{2} Y_{2}-\cdots-D_{r} X_{m} Y_{m}+D_{r} \\
=-D_{r} I_{m}+D_{r}=0_{m}
\end{gathered}
$$

Hence showing that a matrix $R$ constructed from a set of $\mathrm{m}$ latent roots and a corresponding set of $\mathrm{m}$ linearly independent right latent vectors is a right solvent of the matrix polynomial $D(\lambda)$.

It can easily be verified that the right latent vector $X_{i}$ of $D(\lambda)$ associated with the latent root $\lambda_{i}$ is a right eigenvector of the right solvent $\mathrm{R}$. Since $\lambda_{i}$ is an eigenvalue of $R$, it follows that the right eigenstructure of $\mathrm{R}$ captures a part of the right latent structure of $D(\lambda)$.

\section{B. Condition of Existence}

From the above construction, the nodal point is the existence of a set of $\mathrm{m}$ linearly independent right latent vectors associated with a set of $\mathrm{m}$ latent roots of $D(\lambda)$. Existence of such a set insures the existence of a right solvent. If we let $\sigma[D(\lambda)]$ denote the set of all latent roots of $D(\lambda)$ and $\sigma\left[R_{i}\right]$ the set of eigenvalues of the right solvent $R_{i}$, then a complete set of right solvents is obtained if we can find $r$ right solvents such that:

$$
\left\{\begin{array}{c}
\bigcup_{i=1}^{r} \sigma\left[R_{i}\right]=\sigma[D(\lambda)] \\
\sigma\left[R_{i}\right] \cap \sigma\left[R_{j}\right]=\emptyset
\end{array}\right.
$$

A complete set of right solvents will then describe completely the latent structure of $D(\lambda)$.

\section{Construction of Left Solvents}

In a similar manner, we will establish that a left solvent $L \in \mathbb{R}^{m \times m}$ can be constructed from a set $\left\{\lambda_{1}, \lambda_{2}, \ldots, \lambda_{m}\right\}$ of $\mathrm{m}$ latent roots and a corresponding set of $\mathrm{m}$ linearly independent left latent (row) vectors $\left\{Y_{1}, Y_{2}, \ldots, Y_{m}\right\}$.

Theorem 3: The mxm matrix $L=P^{-1} \Lambda P$, where $P=\left(\begin{array}{c}Y_{1} \\ Y_{2} \\ \vdots \\ Y_{m}\end{array}\right), \quad \Lambda=\operatorname{diag}\left(\lambda_{1}, \lambda_{2}, \ldots, \quad \lambda_{m}\right)$ and $P^{-1}=$ $\left(\begin{array}{llll}X_{1} & X_{2} & \cdots & X_{m}\end{array}\right)$ is a left solvent of $\mathrm{D}(\lambda)$.

Proof 3: we have $\sum_{i=1}^{m} X_{i} Y_{i}=I_{m}$ and $X_{i} Y_{j}=\delta_{i j}$ for $\mathrm{i}, \mathrm{j}=$ 1...m.

Evaluating $D(L)=L^{r}+L^{\mathrm{r}-1} D_{1}+\cdots+L D_{r-1}+D_{r}$ leads to

$$
D(L)=P^{-1} \Lambda^{r} P+P^{-1} \Lambda^{r-1} P D_{1}+\cdots+P^{-1} \Lambda P D_{r-1}+D_{r}
$$

or

$$
\begin{gathered}
D(L)=\sum_{i=1}^{m} \lambda^{r} X_{i} Y_{i}+\left(\sum_{i=1}^{m} \lambda^{r-1} X_{i} Y_{i}\right) D_{1} \\
+\cdots+\left(\sum_{i=1}^{m} \lambda X_{i} Y_{i}\right) D_{r-1}+D_{r}
\end{gathered}
$$

$$
\begin{gathered}
D(L)=X_{1}\left[\lambda_{1}^{r} Y_{1}+\lambda_{1}^{r-1} Y_{1} D_{1}+\cdots+\lambda_{1} Y_{1} D_{r-1}\right]+ \\
X_{2}\left[\lambda_{2}^{r} Y_{2}+\lambda_{2}^{r-1} Y_{2} D_{1}+\cdots+\lambda_{2} Y_{2} D_{r-1}\right]+\cdots+ \\
X_{m}\left[\lambda_{m}^{r} Y_{m}+\lambda_{m}^{r-1} Y_{m} D_{1}+\cdots+\lambda_{m} Y_{m} D_{r-1}\right]+D_{r}
\end{gathered}
$$

From $Y_{i} D\left(\lambda_{i}\right)=\theta(i=1 \ldots \mathrm{m})$ it follows that

$$
\lambda_{i}^{r} Y_{i}+\lambda_{i}^{r-1} Y_{i} D_{1}+\cdots+\lambda_{i} Y_{i} D_{r-1}=-Y_{i} D_{r}
$$

and

$$
\begin{gathered}
D(L)=-X_{1} Y_{1} D_{r}-X_{2} Y_{2} D_{r}-\cdots-X_{m} Y_{m} D_{r} \\
=-I_{m} D_{r}+D_{r}=0_{m}
\end{gathered}
$$

Indicating that $\mathrm{L}$ is indeed a left solvent of $D(\lambda)$.

Here again, we can verify that the left latent vector of $D(\lambda)$ associated with the latent root $\lambda_{i}$ is a left eigenvector of the left solvent L. Since $\lambda_{i}$ is an eigenvalue of $L$, it follows that the left eigenstructure of $\mathrm{L}$ captures a part of the left latent structure of $D(\lambda)$.

\section{Condition of Existence}

Just as for the right solvent, the existence of a left solvent depends on the existence of a set of m linearly independent left latent vectors. A complete set of left solvents (covering totally the latent structure of $D(\lambda)$ ) is obtained if we can find $\mathrm{r}$ left solvents where each solvent involves a distinct set of $\mathrm{m}$ latent roots of $D(\lambda)$. This in turn requires that for each such a distinct set, we can find a corresponding set of linearly left latent vectors.

\section{E. Illustrative Example}

Consider

$$
\begin{aligned}
D(\lambda)= & \left(\begin{array}{ll}
1 & 0 \\
0 & 1
\end{array}\right) \lambda^{3}+\left(\begin{array}{ll}
0 & 1 \\
0 & 5
\end{array}\right) \lambda^{2} \\
& +\left(\begin{array}{cc}
-1 & 5 \\
0 & 6
\end{array}\right) \lambda+\left(\begin{array}{ll}
0 & 4 \\
0 & 0
\end{array}\right)
\end{aligned}
$$

where the latent roots are $\{0,1,-1,-2,-3\}$ with 0 being a double root. The right latent vectors corresponding to these latent roots are respectively:

$$
\left\{\left(\begin{array}{l}
1 \\
0
\end{array}\right),\left(\begin{array}{l}
1 \\
0
\end{array}\right),\left(\begin{array}{l}
1 \\
0
\end{array}\right),\left(\begin{array}{c}
1 \\
-3
\end{array}\right),\left(\begin{array}{c}
1 \\
-12
\end{array}\right)\right\}
$$

Each right solvent being a $2 \times 2$ matrix, we form right solvents by pairing latent roots with corresponding linearly independent right latent vectors such as $\{0,-2\},\{0,-3\},\{1$, $-2\},\{1,-3\},\{-1,-2\},\{-1,-3\}$ or $\{-2,-3\}$.

The right solvent, say involving the pair $\{1,-2\}$ is then

$$
R=\left(\begin{array}{cc}
1 & 1 \\
0 & -3
\end{array}\right)\left(\begin{array}{cc}
1 & 0 \\
0 & -2
\end{array}\right)\left(\begin{array}{cc}
1 & 1 \\
0 & -3
\end{array}\right)^{-1}=\left(\begin{array}{cc}
1 & 1 \\
0 & -2
\end{array}\right)
$$

It can be checked that $R^{3}+D_{1} R^{2}+D_{2} R+D_{3}=\left(\begin{array}{ll}0 & 0 \\ 0 & 0\end{array}\right)$

We cannot have in this case a complete set of right solvents since out of the seven possible right solvents, we cannot obtain three disjoint spectra covering the whole spectrum of $D(\lambda)$.

Left latent vectors associated with the latent roots are

$$
\left\{\left(\begin{array}{ll}
0 & 1
\end{array}\right),\left(\begin{array}{ll}
-6 & 5
\end{array}\right),\left(\begin{array}{ll}
1 & 0
\end{array}\right),\left(\begin{array}{ll}
0 & 1
\end{array}\right),\left(\begin{array}{ll}
0 & 1
\end{array}\right)\right\}
$$


The left solvent involving, say, the latent root $\{1,-2\}$ is then:

$$
L=\left(\begin{array}{cc}
-6 & 5 \\
0 & 1
\end{array}\right)^{-1}\left(\begin{array}{cc}
1 & 0 \\
0 & -2
\end{array}\right)\left(\begin{array}{cc}
-6 & 5 \\
0 & 1
\end{array}\right)=\left(\begin{array}{cc}
1 & -2.5 \\
0 & -2
\end{array}\right)
$$

\section{EXPANSION OF $\mathrm{D}^{-1}(\mathrm{~s})$}

In this section we will show that we can determine the inverse of a matrix polynomial $D(\lambda)$ from the block partial fraction expansion of the transfer function of a system described by a right or left matrix fraction description (1).

\section{A. Left $M F D$}

Consider the matrix transfer function of the system given in left matrix fraction description:

$$
\begin{gathered}
G(s)=\left[I s^{r}+D_{1} s^{r-1}+\cdots+D_{r-1} s+D_{r}\right]^{-1} \\
{\left[N_{0} s^{r-1}+N_{1} s^{r-2}+\cdots+N_{r-1}\right]}
\end{gathered}
$$

And $\left\{L_{1}, L_{2}, \ldots, L_{r}\right\}$ a complete set of left solvents of $D(s)$. It can be verified that the state space equation of this system in block observer form is the following:

$$
\left\{\begin{array}{c}
\dot{x}_{o}=A_{o} x_{o}+B_{o} u \\
y=C_{o} x_{o}
\end{array}\right.
$$

where

satisfies

$$
\left\{\begin{array}{c}
A_{o}=\left(\begin{array}{cccc}
0_{m} & \cdots & 0_{m} & -D_{r} \\
I_{m} & \cdots & 0_{m} & -D_{r-1} \\
\vdots & \ddots & \vdots & \vdots \\
0_{m} & \cdots & I_{m} & -D_{1}
\end{array}\right) \\
B_{o}=\left(\begin{array}{c}
N_{r-1} \\
N_{r-2} \\
\vdots \\
N_{0}
\end{array}\right) \\
C_{o}=\left(\begin{array}{llll}
0_{m} & \cdots & 0_{m} & I_{m}
\end{array}\right)
\end{array}\right.
$$

$$
G(s)=C_{o}\left(s I-A_{o}\right)^{-1} B_{o}
$$

Using the transformation $x_{d}=V_{L} x_{o}$ with $\mathrm{V}_{\mathrm{L}}$ is the left block Vandermonde matrix, which is assumed non-singular, we obtain:

$$
\left\{\begin{array}{c}
\dot{x}_{d}=A_{d} x_{d}+B_{d} u \\
y=C_{d} x_{d}
\end{array}\right.
$$

where $\left\{\begin{array}{c}A_{d}=V_{L} A_{o} V_{L}^{-1} \\ B_{d}=V_{L} B_{o} \\ C_{d}=C_{o} V_{L}^{-1}\end{array}\right.$ with

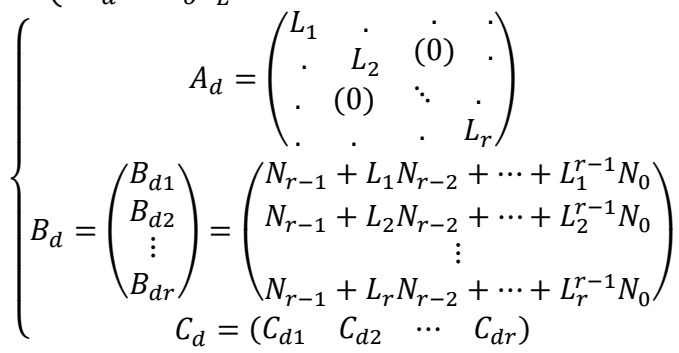

$C_{L}$ is the last block row of $V_{L}^{-1}$.

Now

$$
G(s)=C_{d}\left(s I-A_{d}\right)^{-1} B_{d}=\sum_{i=1}^{r} C_{d i}\left(s I-L_{i}\right)^{-1} B_{d i}
$$

Hence a block partial fraction expansion of $\mathrm{G}(\mathrm{s})$ about a complete set of left solvents is obtained.

A particular case of interest is obtained if: $N_{0}=N_{1}=\ldots=$ $N_{r-2}=0$ and $N_{r-1}=I$, in which case we obtain the decomposition of $D^{-1}(s)$.

Since $B_{\mathrm{o}}=\left(\begin{array}{c}I_{m} \\ 0_{m} \\ \vdots \\ 0_{m}\end{array}\right)$

Then

$B_{d}=V_{L} B_{o}=\left(\begin{array}{cccc}I_{m} & L_{1} & \cdots & L_{1}^{r-1} \\ I_{m} & L_{2} & \cdots & L_{2}^{r-1} \\ \vdots & \vdots & \vdots & \vdots \\ I_{m} & L_{r} & \cdots & L_{r}^{r-1}\end{array}\right)\left(\begin{array}{c}I_{m} \\ 0_{m} \\ \vdots \\ 0_{m}\end{array}\right)=\left(\begin{array}{c}I_{m} \\ I_{m} \\ \vdots \\ I_{m}\end{array}\right)$

and $C_{L}$ is the last block row of $V_{L}^{-1}$.

Hence

$$
D^{-1}(s)=\sum_{i=1}^{r} C_{L_{i}}\left(s I-L_{i}\right)^{-1}
$$

Theorem 4: The inverse of a matrix polynomial $D(\lambda)$ is given by (36), if $D(\lambda)$ presents a complete set of left solvents.

Proof 4: the non-signularity of the left block Vandermonde matrix $V_{L}$ is verified if a complete set of left solvents exist.

\section{B. Right $M F D$}

In a similar manner we can determine $D^{-1}(s)$ from a right MFD of a matrix transfer function. We consider a matrix transfer function as follows:

$$
\begin{array}{r}
G(s)=\left[N_{0} s^{r-1}+N_{1} s^{r-2}+\cdots+N_{r-1}\right] \\
{\left[I s^{r}+D_{1} s^{r-1}+\cdots+D_{r-1} s+D_{r}\right]^{-1}}
\end{array}
$$

With $\left\{R_{1}, R_{2}, \ldots, R_{r}\right\}$ a complete set of right solvents of $D(s)$.

The corresponding state space equation in block controller form is:

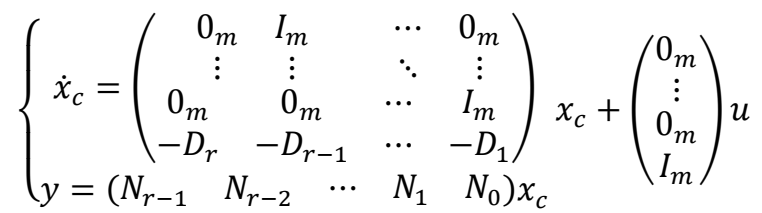

Satisfies $(s)=C_{c}\left(s I-A_{c}\right)^{-1} B_{c}$.

Using the transformation $x_{c}=V_{R} x_{d}$ with $\mathrm{V}_{\mathrm{R}}$ is an assumed non-singular "right" blockVandermonde matrix, we obtain:

$$
\left\{\begin{array}{c}
\dot{x}_{d}=A_{d} x_{d}+B_{d} u \\
y=C_{d} x_{d}
\end{array}\right.
$$

where $\left\{\begin{array}{c}A_{d}=V_{R}^{-1} A_{c} V_{R} \\ B_{d}=V_{R}^{-1} B_{c} \\ C_{d}=C_{c} V_{R}\end{array}\right.$ with

$$
\left\{\begin{array}{c}
A_{d}=\left(\begin{array}{cccc}
R_{1} & \cdot & \cdot & \cdot \\
\cdot & R_{2} & (0) & \cdot \\
\cdot & (0) & \ddots & \cdot \\
\cdot & \cdot & \cdot & R_{r}
\end{array}\right), B_{d}=\left(\begin{array}{c}
B_{d 1} \\
B_{d 2} \\
\vdots \\
B_{d r}
\end{array}\right) \\
C_{d}=\left(\begin{array}{c}
C_{d 1} \\
C_{d 2} \\
\vdots \\
C_{d r}
\end{array}\right)^{T}=\left(\begin{array}{c}
N_{r-1}+N_{r-2} R_{1}+\cdots+N_{0} R_{1}^{r-1} \\
N_{r-1}+N_{r-2} R_{2}+\cdots+N_{0} R_{2}^{r-1} \\
\vdots \\
N_{r-1}+N_{r-2} R_{r}+\cdots+N_{0} R_{r}^{r-1}
\end{array}\right)
\end{array}\right.
$$

$B_{d}$ is the last block column of $V_{R}^{-1}$. 
It follows that $G(s)=C_{R}\left(s I-A_{R}\right)^{-1} B_{R}=\sum_{i=1}^{r} C_{R_{i}}(s I-$ $\left.R_{i}\right)^{-1} B_{R_{i}}$

Leading to a block partial fraction expansion of $G(s)$ about a complete set of right solvents.

If $N_{0}=N_{1}=\ldots=N_{r-2}=0$ and $N_{r-1}=I$, the decomposition of $\mathrm{D}^{-1}(\mathrm{~s})$ follows.

Since $\quad C_{c}=\left(\begin{array}{llll}I_{m} & 0_{m} & \cdots & 0_{m}\end{array}\right)$ and $\mathrm{V}_{\mathrm{R}}$ is the right Vandermonde matrix (as given in (9)), then $C_{R}=C_{c} V_{R}=$ $\left(\begin{array}{lll}I_{m} & \cdots & I_{m}\end{array}\right)$

And $B_{R}$ is the last block column of $V_{R}^{-1}$.

It follows that:

$$
D(s)=\sum_{i=1}^{r}\left(s I-R_{i}\right)^{-1} B_{R_{i}}
$$

Theorem 5: The inverse of a matrix polynomial $D(\lambda)$ is given by (41) iff $D(\lambda)$ presents a complete set of right solvents.

Proof 5: The condition for the previous development to be possible is the fact that the right block Vandermonde matrix $V_{R}$ is non-singular, and this is verified if a complete set of right solvents exist.

\section{Illustrative Example}

Let

$$
D(s)=I s^{2}+\left(\begin{array}{cc}
-1 & 1 \\
0 & -1
\end{array}\right) s+\left(\begin{array}{cc}
0 & -1 \\
0 & -2
\end{array}\right)
$$

with the following left solvents:

$$
L_{1}=\left(\begin{array}{cc}
1 & 0 \\
0 & -1
\end{array}\right), L_{2}=\left(\begin{array}{ll}
0 & 1 \\
0 & 2
\end{array}\right)
$$

Hence

$$
V_{L}=\left(\begin{array}{ll}
I & L_{1} \\
I & L_{2}
\end{array}\right) \Rightarrow V_{L}^{-1}=\left(\begin{array}{cccc}
0 & 1 / 3 & 1 & -1 / 3 \\
0 & 2 / 3 & 0 & 1 / 3 \\
1 & -1 / 3 & -1 & 1 / 3 \\
0 & -1 / 3 & 0 & 1 / 3
\end{array}\right)
$$

it follows that:

$$
\begin{aligned}
& D^{-1}(s)=C_{L_{1}}\left(s I-L_{1}\right)^{-1}+C_{L_{2}}\left(s I-L_{2}\right)^{-1} \\
& D(s)=\left(\begin{array}{cc}
1 & -1 / 3 \\
0 & -1 / 3
\end{array}\right)\left(\begin{array}{cc}
s-1 & 0 \\
0 & s+1
\end{array}\right)^{-1} \\
& +\left(\begin{array}{cc}
-1 & 1 / 3 \\
0 & 1 / 3
\end{array}\right)\left(\begin{array}{cc}
s & -1 \\
0 & s-2
\end{array}\right)^{-1} \\
& \text { Then } D^{-1}(s)=\left(\begin{array}{cc}
\frac{1}{s(s-1)} & \frac{-1}{s(s+1)(s-2)} \\
0 & \frac{1}{(s+1)(s-2)}
\end{array}\right)
\end{aligned}
$$

\section{CONClusion}

Solvents or block roots of a matrix polynomial can be constructed from latent roots and latent vectors. Conditions of their existence are discussed. The block partial fraction expansion of a matrix transfer function expressed as a matrix fraction description is obtained. From this expansion, the inverse of a matrix polynomial is determined and it involves the knowledge of a complete set of solvents and the computation of the inverse of a block Vandermonde matrix. In control theory, dealing with systems represented in matrix fraction description requires often a full set of solvents, and for the case of the block Vandermonde matrix inversion, a recursive algorithm has been elaborated. The importance of solvents or block roots (blocks poles and block zeros) in control systems design stems from the fact that block pole assignment is equivalent to eigenstructure assignment (eigenvalues and eigenvectors) not merely the assignment of poles. This research work is a part of a thesis work on the design of compensators for systems described by matrix fraction descriptions.

\section{REFERENCES}

[1] I. Bras and T. P. de Lima, "A spectral approach to polynomial matrcies solvents," Appl. Math. Lett., vol. 9, no. 4, pp. 27-33, 1996.

[2] V. N. Kublanovskaya, "Methods and algorithms of solving spectral problems for polynomial and rational matrices," J. Math. Sci., vol. 96, no. 3, pp. 3085-3287, 1999.

[3] K. Hariche and E. D. Denman, "On solvents and lagrange interpolating lambda-matrices," App. Math. and Comp., vol. 25, no. 4, pp. 321-332, 1988

[4] S. Zhang, "Inversion of polynomial matrices," Int. J. Control, vol. 46, pp. 33-37, 1987.

[5] A. Schuster and P. Hippe, "Inversion of polynomial matrices by interpolation," IEEE Trans. Autom. Control, vol. 37, pp. 363-365, 1992.

[6] P. Stefanidis, A. P. Paplinski, and M. J. Gibbard, "Numerical operations with polynomial matrices," Lecture Notes in Control and Information Sciences, vol. 171, Springer, Berlin, 1992.

[7] J. C. Basilio, "Inversion of polynomial matrices via state-space," Linear Algebra Appl., vol. 357, pp. 259-271, 2002.

[8] N. P. Karampetakis and P. Tzekis, "On the computation of the generalized inverse of a polynomial matrix," IMA J. Math. Control. Inform., vol. 18, pp. 83-97, 2001.

[9] L. F. A. Pereira, J. F. Haffner, and L. F. Chaves, "Compensator design in the frequency domain using optimization," IECONO2, vol. 2, pp. 1463-1467, 2002.

[10] J. L. Ramos, "A new method for block partial fraction expansion of matrix fraction descriptions," IEEE Trans. Auto. Cont., vol. 36, no. 12, pp. 1482-1485, 1991.

[11] V. R. Dehkordi and B. Boulet, "Robust controller order redeuction," Int. J. Cont., vol. 84, no. 5, pp. 985-997, 2011.

[12] A. Leva and L. Bascetta, "On the design of the feedforward compensator in two-degree-of-freedom controllers," Mechatronics, vol. 16, pp. 533-546, 2006.

[13] E. Periera, "On solvents of matrix polynomials," Appl. Numer. Math., vol. 47, pp. 197-208, 2003.

[14] P. Lancaster and F. Tisseur, "Hermitian quadratic matrix polynomials: Solvents and inverse problems," MIMS Eprint 2010.10, Manchester Institute for Mathematical Sciences, University of Manchester, UK, 2010.

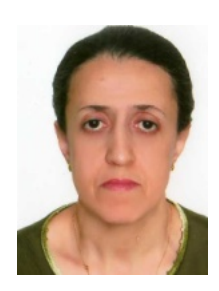

Malika Yaici is a $\mathrm{PhD}$ student at the Institute of Electronics and Electro-techniques of the University of Boumerdes, Algeria. She works on compensators design for MIMO systems. She is also an associate lecturer at the Computer Department of the University of Bejaia, Algeria, where she teaches parallel and distributed algorithms, operating systems and network specification. Her major fields includes distributed systems, control theory, distributed applications, matrix algebra. The paper is part of the thesis research work under the direction of Professor Kamel Hariche.

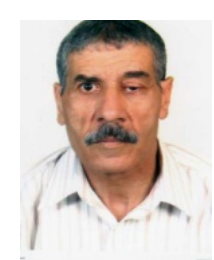

Kamel Hariche received a B.S. degree in physics from the University of Algiers, Algeria (1973), a master's degree in 1978. And a PhD degree in electrical engineering from the University of Houston (Texas-USA). Currently, he is a professor in electrical engineering at the Institute of Electronics and Electro-techniques of the University of Boumerdes, Algeria. He wrote numerous papers in control systems and applied mathematics. 